\title{
THE PROTHROMBIN RESPONSE TO THE PARENTERAL ADMINIS TRATION OF LARGE DOSES OF VITAMIN K IN SUBJECTS WITH NORMAL LIVER FUNCTION AND IN CASES OF LIVER DISEASE: A STANDARDIZED TEST FOR THE ESTIMATION OF HEPATIC FUNCTION ${ }^{1}$
}

\author{
By PAUL N. UNGER AND SHEPARD SHAPIRO, wITH THE \\ TECHNICAL ASSISTANCE OF SHIRLEY SCHWALB \\ (From the Third [New York University] Division, Goldwater Memorial Hospital, \\ Welfare Island, and the Department of Medicine, New York University \\ College of Medicine, New York City)
}

(Received for publication June 26, 1947)

There are a multiplicity of tests for liver functions, each depending upon a particular function of the liver. There has been growing recognition that the response in prothrombin time to the administration of vitamin $\mathrm{K}$ is of aid in the evaluation of hepatic function. The procedures heretofore employed to estimate the prothrombin response have lacked uniformity and, as a consequence, conflicting opinions of its significance and reliability have been expressed. Need exists for further study in both normal and abnormal subjects, and comparison with other liver function tests to establish its status as an index of at least 1 particular function of the liver. The present study deals with a standardized, sensitive method for the estimation of the prothrombin response to the administration of vitamin $\mathrm{K}$. Our results show that the ability to maintain a normal prothrombin level is a function of the liver apt to be affected before others are sufficiently disturbed to permit clinical detection. For this reason, the response of the liver in prothrombin production to the parenteral administration of large doses of synthetic vitamin $K$ is a sensitive indicator of hepatic function.

The use of plasma prothrombin levels as an indicator of the ability of the liver to elaborate prothrombin was originally studied by Stewart and Rourke (1) and pursued subsequently by numerous investigators, (2 to 5), all of whom employed whole plasma for prothrombin assay. Warner, Brinkhous and Smith (6) in dogs and Pohle and Stewart (3) in man, introduced the response of depressed production levels to orally administered

1 Aided by grants from the Blood Transfusion Association of New York and the Hoffman-La Roche Company. vitamin $\mathrm{K}$ derivatives as a test of liver function. They demonstrated that the prothrombin level sometimes failed to return to normal after the ingestion of vitamin $K$. In a few cases, the prothrombin level became even further reduced following the vitamin $\mathrm{K}$ ingestion. The results of these studies need reinvestigation in the light of recent findings that the prothrombin assay techniques utilized were not sufficiently sensitive to detect fine alterations $(7,8)$.

In diseases such as biliary obstruction or diarrhea interfering with the absorption of vitamin $\mathrm{K}$, it cannot be easily decided whether an accompanying hypoprothrombinemia is referable to liver insufficiency or to failure to absorb the vitamin. Kark and Souter (9) showed that patients with hepatic disease having prothrombin deficiency are not able to increase their prothrombin level to normal following parenteral administration of 2methyl-1, 4-naphthoquinone. This was demonstrated, also, by Andrus and Lord (10) using 2 mgm. doses of the substance intramuscularly, and by Seligman, Hurwitz and co-workers (4) using 10 to $20 \mathrm{mgm}$. doses intravenously of synthetic vitamin $\mathrm{K}$ or the sodium salt of its sulphuric ester. It is interesting to note that although Seligman and Hurwitz (4) failed to comment on it, some of their patients with liver disease also exhibited further reduction in the prothrombin level after the medication. In these studies, also, the fact that the methods used were, for the most part, too insensitive to reveal alterations in plasma prothrombin levels of low magnitude needs emphasis (7, 8).

Introduction of the diluted (12.5 per cent) plasma method of estimation of prothrombin time 
greatly increased the sensitivity of the procedure $(7,8)$. Subsequently, Shapiro and Richards $(11,12)$ reported on the plasma prothrombin levels and the response to parenteral administration of synthetic vitamin $\mathrm{K}$ (Hykinone) in liver disease using this procedure. In experiments on dogs, in which liver damage had been produced by the administration of $\mathrm{CCl}_{4}$, it was found that the prothrombin level of the diluted (12.5 per cent) plasma was at least as frequently an indicator of hepatic disturbance as bromsulphalein retention. Their studies of 23 cases of liver disease in man, revealed moderate or marked reduction of the resting prothrombin level in 20 cases in which the reduced prothrombin level failed to return to normal following the intravenous administration of Hykinone. In some cases, moreover, a temporary reduction of a previously normal prothrombin level to abnormal levels occurred. In other patients, further depression of a previously low prothrombin level occurred.

The present study, presented in its earlier stages before the New York Pathological Society (13), is an extension of the study of Shapiro and Richards $(11,12)$ embodying the principle of imposing a load on the liver by the parenteral administration of large doses of vitamin $K$. It is the same principle that is utilized in other liver function tests such as bromsulphalein retention, bilirubin clearance, glucose tolerance, and galactose tolerance tests. This reaction to injection of vitamin $\mathrm{K}$ is correlated in patients with hepatic disease and in normal controls with the results of other liver function tests and liver biopsy or postmortem findings wherever possible. Evidence is presented that the sensitivity of this test for a specific known physiologic function of the liver makes its incorporation into a battery of liver function tests highly desirable.

\section{PROCEDURE}

The resting level of prothrombin was obtained by the diluted (12.5 per cent) plasma method on several days prior to, and on the day of the initial intravenous administration of synthetic vitamin $\mathrm{K}$ (Tetrasodium 2-methyl-1, 4-naphthohydroquinone diphosphoric acid ester [Synkayvite] ${ }^{2}$ ). Normal plasma prothrombin time of diluted (12.5 per cent) plasma was 39.5 seconds \pm 2.5 seconds

2 Synkayvite supplied by Hoffman-La Roche Company, Nutley, N. J.
$(7,8)$. Whole plasma prothrombin times, estimated simultaneously, are not reported in the present study because the methods lack sensitivity and fail to eliminate the effect of natural anticoagulants and interfering substances, and because the results were not of adequate significance. At the beginning of the investigation, $38 \mathrm{mgm}$. of synthetic vitamin $\mathrm{K}$ (Synkayvite) was given intravenously on 4 successive days after the resting level had been determined. Later in the study, the daily dose was increased to $76 \mathrm{mgm}$. of Synkayvite. This is the dose now used. The prothrombin time was then estimated daily during the course of the test period and for several days following the last dose of vitamin $\mathrm{K}$. Estimations were made in duplicate and preferably on freshly drawn fasting samples. Lipemic plasmas interfere with the accuracy of the prothrombin estimation (14).

In all subjects the results of the vitamin $K$ tolerance test were correlated wherever feasible with bromsulphalein retention, cholesterol and esters, albumin and globulin, cephalin flocculation, icteric index and Van den Bergh reaction. There were 2 periods during the course of this study when bromsulphalein was withdrawn from use for investigation by the manufacturers, because of marked reactions which followed its administration. No untoward reaction to the dose of Synkayvite employed appeared in any of the subjects studied.

The methods used in performing the other liver function tests in this study were standard procedures. The bromsulphalein tests were made by administering $5 \mathrm{mgm}$. of bromsulphalein per kilogram of body weight, and taking a 5- and 30-minute blood specimen. Any retention of over 10 per cent bromsulphalein at the end of $1 / 2$ hour was considered abnormal. The cholesterol and ester determinations were made using the Bloor technique. Cephalin flocculation tests were performed using Difco Cephalin and reading at the end of 24 and 48 hours.

\section{RESULTS}

One hundred and thirteen tests were made on 110 individuals. Of these, 47 vitamin $\mathrm{K}$ tolerance tests were interpreted as negative, 9 doubtful and 57 positive.

Negative tests: A negative test is defined as one in which there is either: (Figure 1A) no significant rise in the prothrombin time from a previously normal resting level (i.e., all values remain within the normal range) following the administration of test doses of synthetic vitamin $K$, (B) a return of prothrombin time from hypoprothrombinemic levels to normal levels, (C) a reduction in prothrombin time from normal levels to hyperprothrombinemic levels. In the negative series the resting level was usually 42 seconds or less, and the maximum prothrombin time reached during the test was 45 seconds or less with return toward 


\section{NEGATIVE TESTS}

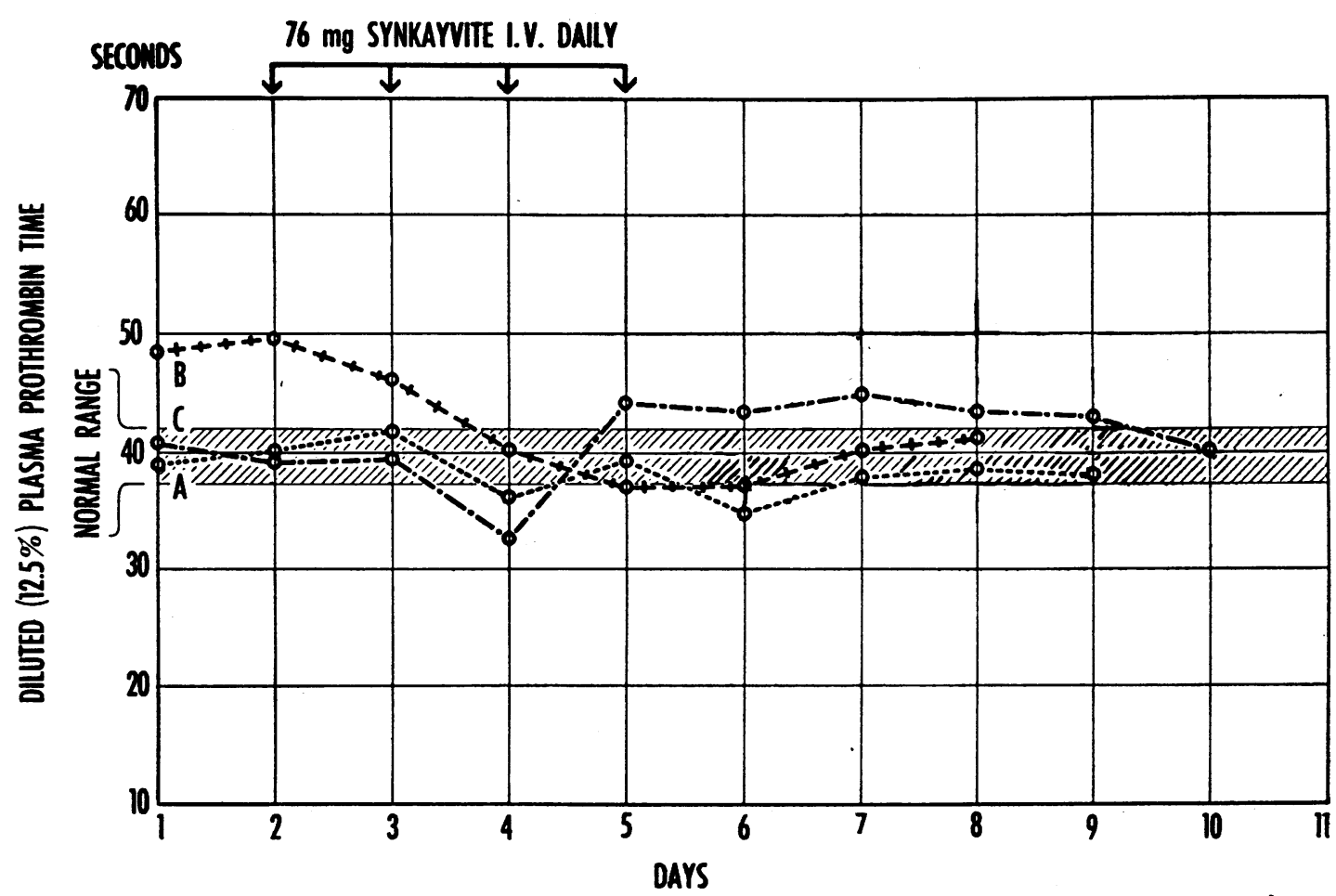

Fig. 1. Three Types of Negative Tests

Curve A remains within the normal range throughout the period of the test. Curve B: initial hypoprothrombinemia with restoration to normal after administration of vitamin $\mathrm{K}$. This is typical of nutritional vitamin $\mathrm{K}$ deficiency. Curve $\mathrm{C}$ : temporary hyperprothrombinemia after the large doses of vitamin $\mathrm{K}$.

the resting level of the prothrombin time following the completion of the test. Of the 47 negative cases 35 started with resting prothrombin times of 42 seconds or less. Twelve cases started with resting times higher than 42 seconds which responded promptly to the parenteral administration of vitamin $\mathrm{K}$ by restoration to normal. This is the typical response seen in vitamin $\mathrm{K}$ deficiency states.

Positive tests: A positive test is defined as one in which there is either (Figure 2A and B) a failure to lower an abnormally prolonged prothrombin time to normal limits during the test period, (C) a further elevation of an already prolonged prothrombin time, or (D) a transient rise in prothrombin time, from a previously normal value to more than 47 seconds in one or more determinations during the test period.

Out of 57, 11 cases started with normal resting prothrombin times (12.5 per cent plasma), and 46 started with abnormal prothrombin times.

In all of these patients, the vitamin $\mathrm{K}$ tolerance test was positive. If the resting prothrombin time had been used alone as a test for liver function, 20 per cent of the cases would have been interpreted as showing no impairment in the hepatic function.

Furthermore some patients with prolonged pretesting times due to deficiency states may have no impairment of liver function. It is realized that it is a somewhat arbitrary limit for bio-assay technique to establish the dividing point between normal and abnormal responses to the administration of vitamin $\mathrm{K}$ at 45 seconds. For this reason, a certain degree of flexibility should be followed in interpreting results several seconds over 45 until further experience shows that this level is completely justifiable. Results between 45 and 47 
POSITIVE TESTS

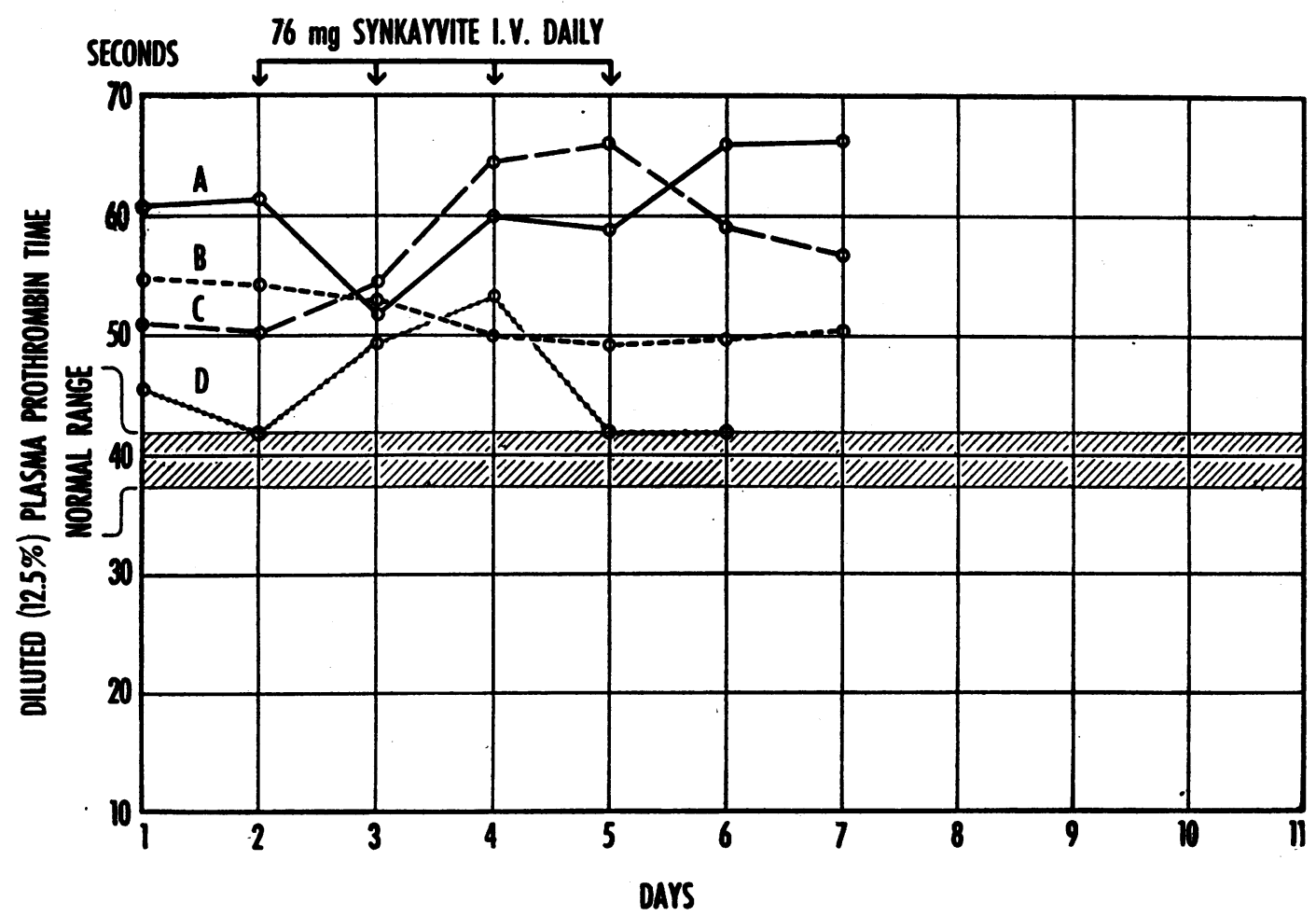

Fig. 2. Positive Tests

Curve A: initial hypoprothrombinemia with partial restoration toward normal following vitamin $\mathrm{K}$ administration followed by further prolongation beyond the resting level. Curve B: initial hypoprothrombinemia unaltered by vita$\min \mathrm{K}$. Curve $\mathrm{C}$ reveals further extension of prothrombin time promptly following vitamin $\mathrm{K}$ administration. Curve $\mathrm{D}$ : normal resting level of prothrombin followed by temporary hypoprothrombinemia after vitamin $\mathrm{K}$ administration and eventual restoration to normal.

seconds have therefore been classified as doubtful.

The type of response to parenteral administration of vitamin $\mathrm{K}$ and/or its degree has not been used to date as an aid in judging prognosis.

\section{ANALYSIS OF RESULTS}

The cases have been arranged from the viewpoint of the presence or absence of liver disease on the basis of the clinical diagnosis. Cases 1 through 36 include a group of subjects, with varying diagnoses, none of whom showed any clinical evidence of liver disease. The maximum prothrombin time reached during the course of the test did not exceed 45 seconds in any of the patients in this group. Two cases, one with a maximum prothrombin time of 44.8 and the other with a maximum prothrombin time of 45.0 , were classified as doubtful. Neither of these 2 cases showed abnormal BSP retention. In the remaining 34 cases in this group of 36 patients, the vitamin $\mathrm{K}$ tolerance test yielded results interpreted as negative. Bromsulphalein retention was tested in 29 of these cases, with less than 10 per cent retention after $1 / 2$ hour in 25 cases, and 10 per cent or more in 4 cases. In the 4 cases where more than 10 per cent of the injected BSP was retained, the largest amount retained was 14 per cent. None of these 4 cases showed clinical evidence of liver disease.

Cases 37 through 48 consist of a group of patients where the maximum prothrombin time reached during the test also did not exceed 45 seconds. The results of the vitamin $\mathrm{K}$ tolerance test were interpreted as negative in these 12 . Included in this group was 1 patient who showed cardiac cirrhosis on postmortem examination. The 
BSP retention and cephalin flocculation tests were normal. Two patients in this group have amyloidosis and both showed negative BSP retention and cephalin flocculation. Another case showed, on postmortem examination, a carcinoma of the tail of the pancreas with a small number of metastases to the liver, too insignificant to have interfered with hepatic function. The BSP retention test was negative, and the cephalin flocculation test was 2 plus. One case was believed to have a fatty liver. This patient showed 14 per cent BSP retention after $1 / 2$ hour, a 4 -plus cephalin flocculation test and a negative vitamin $\mathrm{K}$ tolerance test. This is the only instance in this group of 12 patients where the vitamin $\mathrm{K}$ tolerance test was negative despite clinical evidence of liver disease as revealed by other liver function tests. One case, despite the absence of clinical evidence of liver disease, showed an abnormal BSP retention, a cephalin flocculation of 2 plus and a negative vitamin $\mathrm{K}$ tolerance test. In summary, in this group of 12 patients, there were 5 with clinical evidence of liver disease in all of whom the vitamin $\mathrm{K}$ tolerance test was negative. In only 1 of these 5 could any evidence of impaired liver function be found using other liver function tests. It seems quite reasonable to assume that the patient with a small amount of metastases to the liver had no impairment of hepatic function. The lack of evidence of impairment of hepatic function in the 2 patients with amyloidosis is not unusual for this disease.

Cases 49 through 56 include a group of 8 cases in whom the maximum prothrombin time reached during the test ranged from 45 to 47 seconds. The vitamin $\mathrm{K}$ tolerance test has been interpreted as doubtful in this group, though there is reason to believe from the clinical diagnoses that the results of the vitamin $\mathrm{K}$ tolerance test probably belong in the positive group. It is apparent that a dividing line between positive and negative vitamin $\mathrm{K}$ tolerance tests establishes itself somewhere within the range of 45 to 47 seconds. For the present, until more data are collected, we will interpret results that fall within this range as doubtful and requiring further investigation. There were 5 cases in this group of 8 patients where there was definite clinical evidence of liver disease with positive BSP tests in 2 of the 5 cases. Two others showed 3-plus cephalin flocculations.

The remainder of the patients, cases 57 to 113 , all showed maximum prothrombin times exceeding 47 seconds. There were 7 patients with maximum prothrombin times ranging from 48.2 to 49.4 seconds. Four of these patients have unequivocal clinical evidence of liver disease. Of the remaining 3 cases, 1 was a patient with chronic congestive failure and persistent hepatomegaly; another was a patient with pernicious anemia; the third was a patient in whom no clinical evidence of liver disease was apparent but who showed both positive BSP and Vitamin $\mathrm{K}$ tolerance tests.

Cases 64 through 99 include a group of 36 cases in whom the maximum prothrombin time reached during the vitamin $\mathrm{K}$ tolerance test ranged from 50 to 60 seconds. Twenty-six of the patients in this group showed definite clinical evidence of liver disease. The remaining 10 patients had no evidence of specific liver disease. Five were severely anemic; 1 suffered from nutritional deficiencies with moderate anemia; and 3 showed no clinical evidence whatsoever of liver disease but were over 75 years of age. The finding of impaired liver function in the aged in the absence of clinical evidence of any specific type of hepatic disease has been reported previously (15). The remaining patient was a member of the hospital staff who was studied for control purposes, and in whom the results of vitamin $K$ tolerance test were abnormal on 2 separate occasions. There was no history of any type of liver disease in this subject although he had served recently in the armed forces in areas where infectious hepatitis was epidemic. The only positive clinical finding was enlargement of the liver, palpable 2 fingers below the costal margin. All other liver function tests were negative, though the icteric index was 11.2 units. We consider this as a false positive vitamin $\mathrm{K}$ tolerance test.

The last group of 14 patients (cases 100 through 113) include those in whom the maximum prothrombin times reached during the course of the test ranged from 60 to 155 seconds. All of these cases disclosed definite clinical evidence of liver disease.

In summary, the 113 cases in whom the vitamin $\mathrm{K}$ tolerance test was correlated with clinical findings and other liver function tests showed the following (Figure 3 ): Of 43 cases with no clinical evidence of liver disease, the vitamin $K$ tolerance test was interpreted as negative in 42 and doubt- 




Fig. 3. The Cases Are Arranged from the Viewpoint of Presence or Absence OF LIVER Disease

In 43 cases without liver disease, none yielded a positive result in the vitamin $\mathrm{K}$ tolerance test ; in 56 cases with hepatic disease only 5 showed a negative result, and in 18 of these cases in which biopsy studies established the presence of hepatic disease, no negative results were obtained. The results of the vitamin $\mathrm{K}$ tolerance tests are correlated with bromsulphalein retention and cephalin cholesterol flocculation.

ful in 1 . In 34 of these cases where BSP retention was tested, 30 showed negative and 4 positive BSP tests. There were 14 other cases without evidence of any specific liver disease. Thirteen of these had conditions known to be associated with impaired liver function and 1 subject had no demonstrable disease. The vitamin $\mathrm{K}$ tolerance test was positive in 12 and doubtful in 2 of these 14 subjects. BSP retention was tested in 11 of these cases with 4 positive and 7 negative results. There were 56 cases with clinical evidence of liver disease, 45 of which showed positive, 6 doubtful and 5 negative vitamin $K$ tolerance tests. $\mathrm{BSP}$ retention was tested in 42 of these cases with 20 positive and 22 negative results. Of the 5 cases with clinical evidence of liver disease and negative vitamin $\mathrm{K}$ tolerance tests, 3 had diseases which do not commonly result in impaired liver function (amyloidosis and small hepatic metastases), 1 with a fatty liver which may be associated with impaired liver function, and the remaining case with a disease (cardiac cirrhosis) which may or may not affect liver function. BSP retention was tested in all 5 of these cases with 4 negative and 1 positive result. From the foregoing analysis of this group of cases it appears that the vitamin $\mathrm{K}$ tolerance test exhibits excellent correlation with other clinical findings indicative of impaired liver function referable to various causes.

\section{Analysis of cases with postmortem, biopsy, or operative findings}

It was possible also to correlate the results of liver function tests, including the vitamin $\mathrm{K}$ tolerance test, with biopsy, postmortem, or operative findings in 24 cases (Figure 3 ).

In summary, 18 of the 24 cases yielded evidence from tissue study of liver disease. Sixteen of these cases showed a positive and 2 a doubtful vitamin $\mathrm{K}$ tolerance test. Thirteen of these 18 cases in which bromsulphalein retention was tested yielded 8 positive and 5 negative results. Four of the 24 patients in whom examination of liver tissue was possible showed no histological evidence of liver disease. In all 4 , vitamin $K$ tolerance and $\mathrm{BSP}$ tests were both negative. In the remaining 
2 cases, 1 of amyloidosis and 1 of cardiac cirrhosis, vitamin $\mathrm{K}$ tolerance and BSP tests were both negative. One case, of Simmonds' disease, is worthy of special comment because of the bizarre nature of the response of the prothrombin level to the parenteral administration of vitamin $K$ (Synkayvite). This patient started with a prolonged resting prothrombin time which returned slowly over a course of 10 days to normal values.

The cephalin-cholesterol flocculation test was performed on 22 patients. Among these there were 17 who showed pathological evidence of liver disease. In 5 the cephalin-cholesterol flocculation test was less than 2 plus, and in 12 it was 2 plus or over. In the 5 cases who exhibited both negative vitamin $\mathrm{K}$ tolerance and BSP tests, there were 3 cases which showed no pathological evidence of liver disease, but yielded a 2 plus cephalincholesterol flocculation test. In 1 case with cardiac cirrhosis, and 1 with amyloidosis, the cephalincholesterol flocculation test was negative.

From this review of cases where pathological examination of the liver was obtained, the vitamin $\mathrm{K}$ tolerance test appears to be a reliable indicator of hepatic disease and impaired hepatic function.

\section{DISCUSSION}

Test of hepatic function as an aid in the diagnosis and prognosis of liver disease is perhaps best served by the use of a number of liver function tests. This is inherent in the fact that the liver serves many functions, has great reserve and regenerative ability (16 to 20$)$. Neefe $(19,20)$ states that the thymol turbidity, cephalin flocculation, and the colloidal gold tests are the most sensitive indicators of persistent hepatic dysfunction in cases of infectious hepatitis. Unfortunately, plasma prothrombin levels after vitamin $\mathrm{K}$ administration were not done. Since the thymol turbidity and cephalin flocculation tests depend upon the presènce of beta and gamma globulins, respectively (21), serum protein changes without hepatic cellular involvement may give rise to positive tests.

Neefe and Rheingold (20) found in infectious hepatitis that bromsulphalein retention and bilirubinuria were usually the first tests to indicate early preicteric hepatitis. The results of the bromsulphalein test are believed to depend upon involvement of the polygonal cells of the liver. This test appears to be of value in the diagnosis of many forms of liver disease. Several factors, however, tend to diminish the reliability of this test. First, bromsulphalein is excreted through the bile passages; hence any lesions, extrinsic or intrinsic, producing biliary tract obstruction will delay the excretion of the dye from the blood stream whether or not it is associated with impaired liver function: Secondly, in conditions of circulatory stasis where complete mixing of injected dye occurs slowly, BSP retention may be abnormal due to circulatory factors rather than hepatic dysfunction. Thirdly, bromsulphalein is a substance foreign to the metabolism of the body. The testing of a normal physiological function would appear to be more desirable.

In the evaluation of any liver function test, its specificity for measuring a particular function is often as important as its reliability and sensitivity. On the basis of the material studied and the analysis of results, the vitamin $\mathrm{K}$ tolerance test appears to be a sensitive index of the ability of the liver to produce prothrombin. The results suggest, further, that this function moves parallel with, and is one of the more delicate indicators of, the general functional state of the liver. In certain instances it is more sensitive than the bromsulphalein retention test. The test has also the distinct advantage that it measures a function which apparently resides only in liver tissue. Prothrombin production is also disturbed in vitamin $\mathrm{K}$ (nutritional) deficiency but these cases respond readily to the parenteral administration of vitamin $\mathrm{K}$ by return of low plasma prothrombin levels to normal. There may be a shift in other components concerned in the clotting mechanism at the same time that prothrombin production becomes reduced. As far as fibrinogen is concerned (22) preliminary studiès (23) indicate that it does not play a role in the changes in prothrombin time elicited by the single-stage technique as employed in the present study in liver disease. Further investigation of the problem is in progress in this laboratory and will be reported.

The further depression of the prothrombin level, or activity following the parenteral administration of large doses of vitamin $K$, suggests that in the cases of hepatic disease studied, the liver appears to be functioning at its maximum capacity under the prevailing conditions. The demonstrated 
striking sensitivity of the mechanism suggests that the hepatic reserve for this function is less than that of other known and measurable liver functions. The prolongation of prothrombin time observed after the administration of large doses of vitamin $\mathrm{K}$ in liver disease may occur either because of inadequate production of the substrate with which vitamin $\mathrm{K}$ combines, or the utilization of this substrate in an abnormal manner and consequent interference with the production of prothrombin. This would reduce the substrate available for prothrombin elaboration. As soon as the substrate is replenished the resting level of prothrombin becomes reestablished.

If the liver stores vitamin $\mathrm{K}$, it seems logical to assume that in hepatic disease this capacity becomes reduced. The normal liver would use its vitamin $\mathrm{K}$ stores as needed, restore its supply as needed and thus maintain the prothrombin activity at a constant level.

Whatever the mechanism, the sudden influx of a large quantity of vitamin $\mathrm{K}$ may tend to accelerate prothrombin production. This is well demonstrated in some normal subjects in which a temporary hyperprothrombinemia occurs following the injection of vitamin $\mathrm{K}$ derivatives in large doses (24). The response of a diseased liver to such stimulation might be reflected in an exhaustion phenomenon with consequent prolongation of the prothrombin time. It is noteworthy that this reaction had been observed in only a certain proportion of persons examined. Some retain a fixed level of prothrombin despite administration of massive doses of vitamin $\mathrm{K}$. This is true of normals as well as cases of liver disease.

In all cases in which existing hypoprothrombinemia was exaggerated by large doses of vitamin $K$, the phenomenon was transitory, lasting only 24 to 48 hours. Nevertheless, it is suggested that in patients with hepatic disease in whom the prothrombin time is already prolonged, that if vita$\min \mathrm{K}$ is used, a small dose be given with repetition only where the prothrombin time does not further increase. It is particularly important where hemorrhage has occurred recently, or where the tendency to bleed is present. The only manner in which prothrombin activity can be augmented in such cases is by means of transfusion, preferably of whole, fresh blood, or possibly by administration of frozen plasma (6). None of the cases in our series exhibited bleeding phenomena.

Although our results indicate that the vitamin $\mathrm{K}$ tolerance test shows a high degree of sensitivity for detecting slight degrees of hepatic functional impairment, the test cannot serve alone to establish the presence or absence of liver disease. It should be correlated with all other means of clinical examination and appropriate function tests to form an inventory, so to speak, of the state of the liver. The value of biopsy study, particularly in doubtful cases, cannot be overestimated as confirmatory evidence.

It is not yet clear that a particular type of response is of prognostic value. Repeated tests in the same patient, in a large series of cases over an extended period of time, and after different types of therapy, may help determine this important point. The simplicity of performance of the vitamin $\mathrm{K}$ tolerance test permits its application on a large scale.

\section{SUMMARY AND CONCLUSIONS}

1. A standardized vitamin $K$ tolerance test estimating the prothrombin response to the parenteral administration of large test doses of vitamin $\mathrm{K}$ has been used.

2. In a series of 57 cases without clinical evidence of liver disease, and 56 cases with clinical evidence of liver disease, the results have been correlated with other liver function tests. Twentyfour of the 113 cases had morphologic study of the liver.

3. The vitamin $\mathrm{K}$ tolerance test exhibits excellent correlation with other clinical findings indicative of impaired liver function referable to various causes.

4. The vitamin $\mathrm{K}$ tolerance test was found to be a sensitive indicator of hepatic function.

5. This test is one of considerable sensitivity for measuring the hepatic function of prothrombin formation and can serve as a good index of the presence or absence of hepatic disease.

The thromboplastin used in these studies was supplied by the Maltine Co., Brooklyn, N. Y.

\section{BIBLIOGRAPHY}

1. Stewart, J. D., and Rourke, G. M., Prothrombin and vitamin K therapy. New Eng. J. Med., 1939, 221, 403. 
2. Allen, J. G., and Julian, O. G., Response of plasma prothrombin to vitamin $\mathrm{K}$ substitute therapy in cases of hepatic disease. Arch. Surg., 1940, 41, 1363.

3. Pohle, F. J., and Stewart, J. K., Observations on plasma prothrombin and effects of vitamin $\mathrm{K}$ in patients with liver or biliary tract disease. J. Clin. Invest., 1940, 19, 365.

4. Seligman, A. M., Hurwitz, A., Frank H. A., and Davis, W. A., The intravenous use of synthetic vitamin K. Surg., Gyn., and Obst., 1941, 73, 686.

5. Allen, J. G., and Julian, O. C., Prothrombin and hepatic function. Arch. Surg., 1942, 45, 691.

6. Smith, H. P., Warner, E. D., and Brinkhous, K. M., Prothrombin deficiency and the bleeding tendency in liver injury (chloroform intoxication). J. Exper. Med., 1937, 66, 801.

7. Campbell, H. A., and Link, K. P., Studies on the hemorrhagic sweet clover disease; isolation and crystallization of hemorrhagic agent. J. Biol. Chem., 1941, 138, 21.

8. Shapiro, S., Sherwin, B., Redish, M., and Campbell, H. A., Prothrombin estimation; a procedure and clinical interpretations. Proc. Soc. Exper. Biol. \& Med., 1942, 50, 85.

9. Kark, R., and Souter, A. W., Synthetic vitamin $\mathrm{K}$ in treatment of hypoprothrombinemia. Lancet, 1940, $1,1149$.

10. Andrus, W. DeW., and Lord, J. W., Jr., The physiology of plasma prothrombin and its relation to liver function. Surgery, 1942, 12, 801.

11. Richards, R. K., and Shapiro, S., Experimental and clinical studies on the action of high doses of hykinone and other menadione derivatives. J. of Pharmacol. \& Exper. Therap., 1945, 84, 93.

12. Shapiro, S., and Richards, R. K., The prothrombin response to large doses of synthetic vitamin $\mathrm{K}$ in liver disease. Ann. Int. Med., 1945, 22, 841.

13. Zimmerman, S. P., Rosenthal, N., Unger, P. N., and
Shapiro, S. Proc. New York Pathological Society (In press).

14. Pohle, F. J., and Stewart, J. K., Study of the Quick method for the quantitative determination of prothrombin with suggested modifications. Am. J. M. Sc., 1939, 198, 622.

15. Rafsky, H. A., and Newman, B., Liver function tests in the aged (serum cholesterol partition, bromsulphalein, cephalin-flocculation and oral and intravenous hippuric acid tests). Am. J. Digest. Dis., 1943, 10, 66.

16. Teitlebaum, M., Curtis, A. C., and Goldhamer, S. M., The comparative value of several liver function tests. Ann. Int. Med., 1945, 22, 653.

17. Svirbely, J. L., Monaco, A. R., and Alford, W. C., The comparative efficiency of various liver function tests in detecting hepatic damage produced in dogs by xylidine. J. Lab. \& Clin. Med., 1946, 31, 1133.

18. Drill, V. A., and Ivy, A. G., Comparative values of bromsulphalein, serum phosphatase, prothrombin time, and intravenous galactose tolerance test in detecting hepatic damage produced by carbon tetrachloride. J. Clin. Invest., 1944, 23, 209.

19. Neefe, J. R., Results of hepatic tests in chronic hepatitis without jaundice. Gastroenterology, 1946, $7,1$.

20. Neefe; J. R., and Rheingold, J. G., Laboratory aids in the diagnosis and management of infectious hepatitis. Gastroenterology, 1946, $7: 393$.

21. Cohen, P. C., and Thompson, F., The serum protein fraction responsible for the thymol turbidity test. J. Lab. \& Clin. Med., 1947, 32, 314.

22. Lyons, R. N., Thiol-vitamin $\mathrm{K}$ mechanism in the clotting of fibrinogen. Australian J. Exper. Biol. \& M. Sc., 1945, 23, 131.

23. Shapiro, S., Unpublished data. Manuscript in preparation.

24. Unger, P. N., and Shapiro, S., Hyperprothrombinemia induced by vitamin $\mathrm{K}$ in human subjects with normal liver function. Blood (In press). 